\section{The effect of cerium oxide on lung injury following lower extremity ischemia- reperfusion injury in rats under desflurane anesthesia}

\author{
Aycan Ozdemirkan, MD, Aysegul Kucuk, MD, \\ Isin Gunes, MD, Mustafa Arslan, MD, \\ Aydin Tuncay, MD, Volkan Sivgin, MD, \\ Saban C. Sezen, MD, Hakan Boyunaga, MD.
}

\section{ABSTRACT}

Objectives: To examine the effects of desflurane and cerium oxide (CO) on lung tissue following ischemiareperfusion injury (IRI).

Methods: Experiments were conducted in Gazi University Animal Laboratory, Ankara, Turkey. Thirty rats were divided into 5 groups: control (C), IRI, IRI-CO, IRI-desflurane (IRID), IRI-CO-desflurane (IRICOD). Cerium oxide was given intraperitoneally. Lower extremity IRI was induced. Desflurane was applied during IRI. Lung histopathological examinations and serum biochemical analyses were performed.

Results: Serum nitricoxide (NO) and malondialdehyde (MDA) levels were higher in group IRI $(p=0.006)$ than in group C $(p=0.001)$. Serum MDA and NO levels were significantly lower in groups IRICO and IRICOD than in group IRI. Significantly greater alveolar wall thickening and neutrophil infiltration were recorded in group IRI than in group C. Coadministration of desflurane and $\mathrm{CO}$ significantly decreased alveolar wall thickening and neutrophil infiltration compared to group IRI. Total lung injury scores were significantly lower in groups IRID, IRICO, and IRICOD than in group IRI.

Conclusion: Intraperitoneal $\mathrm{CO}$ with desflurane, reduced oxidative stress and corrected the damage in lung. Cerium oxide given before and desflurane given during IRI have been shown to have protective effects on lung damage in rats.

Keywords: cerium, desflurane, ischemia-reperfusion injury, lung injury

Saudi Med J 2021; Vol. 42 (11): 1247-1251 doi: 10.15537/smj.2021.42.11.20210104

Tschemia-reperfusion injury (IRI) caused by redirecting of blood flow to an ischemic tissue involves the ischemic organ and distant organs, and ultimately causes multiorgan failure. Lower limb IRI is known to induce remote organ dysfunctions. ${ }^{1}$ The lungs are particularly vulnerable to IRI, as they have the first major vasculature exposed to ischemic blood. ${ }^{2}$ The recognition that reactive oxygen species (ROS) play an important role in IRI-related tissue damage has made oxidative stress and mitochondrial dysfunction the main targets in treatment of lower limb IRI. ${ }^{1}$ Several agents have been studied to reduce oxidative damage in various organs. ${ }^{3}$ In particular, beneficial effects of isoflurane and sevoflurane have been shown in IRI-induced lung injury. However, conflicting results have been reported regarding the effects of desflurane against lung damage following IRI. ${ }^{3,4}$ Pathologies associated with oxidative damage respond favorably to compounds that can scavenge ROS. One of them is cerium oxide (CO), a nanomaterial under investigation by the US Environmental Protection Agency. With its scavenging properties of radical oxygen species, its efficacy has been demonstrated experimentally in oxidative damage. ${ }^{5,6}$ While it has been shown that $\mathrm{CO}$ reduces oxidative damage, conflicting results about desflurane are available in the literature. ${ }^{4-6}$ In our previous study, the combined effects of sevoflurane and $\mathrm{CO}$ on lung injury were investigated and it was revealed that they showed a stronger lung protective effect than their individual effects. $^{7}$ In this study, we aimed to study the combined effects of desflurane and $\mathrm{CO}$ on lung tissue following lower extremity IRI in rats.

Methods. Procedures were approved by Gazi University Ethical Committee of Experimental Animals (G.Ü.ET-19.043) and carried out in Gazi University Animal Laboratory in accordance with the Guide for the Care and Use of Laboratory Animals, in Ankara, Turkey. Experiments were performed on 30 male 8-10 months old Wistar Albino rats weighing 250-300 g during daylight hours. Animals were housed under identical environmental conditions and kept at a neutral temperature $\left(20-21^{\circ} \mathrm{C}\right)$ under a $12: 12$ hour photoperiod. Food and water were available ad libitum.

Experimental groups. Thirty rats were randomized into 5 groups ( $\mathrm{n}=6$ per group). Group 1: control group (C), Group 2: ischemia-reperfusion injury group (IRI), Group 3: cerium oxide-IRI group (IRICO), Group 4:

Disclosure. Authors have no conflict of interests, and the work was not supported or funded by any drug company. 
IRI-desflurane group (IRID), Group 5: cerium oxideIRI-desflurane group (IRICOD)

Experimental protocols. $50 \mathrm{mg} / \mathrm{kg}$ ketamine hydrochloride (VetaKetam; Vetagro, Poland) and $20 \mathrm{mg} / \mathrm{kg}$ xylazine (XYLAZINBIO \%2- Bioveta, Czech Republic) were administered intraperitoneally (IP) for anesthesia. Intraperitoneal injection was preferred because it allows rapid absorption.

Group C: midline laparotomy was performed. Group IRI: midline laparotomy was performed, followed by 120 minutes of ischemia by clamping the infrarenal abdominal aorta by placing an atraumatic microvascular clamp, and then 120 minutes of reperfusion. ${ }^{7}$

Group IRICO: $0.5 \mathrm{mg} / \mathrm{kg}$ IP cerium oxide (SigmaAldrich, Co. St. Louis, MO, USA) was administered. Midline laparotomy was performed 30 minutes following the administration of $\mathrm{CO}$, followed by 120 minutes ischemia and 120 minutes of reperfusion.

Group IRID: Midline laparotomy and IRI were performed, and desflurane (Suprane, Baxter, IL, USA) was applied at a $6 \%$ concentration and a rate of $4 \mathrm{~L} / \mathrm{min}$ in $100 \% \mathrm{O}_{2}$ concurrently with the onset of the ischemia period and extending for 4 hours. Desflurane inhalation was carried out in a $40 \times 40 \times 70 \mathrm{~cm}$ transparent box.

Group IRICOD: Intraperitoneal CO $(0.5 \mathrm{mg} / \mathrm{kg})$ was administered. Laparotomy was performed 30 minutes following $\mathrm{CO}$. Ischemia-reperfusion injury and desflurane were administered as per Group IRID.

At the end of the experiments, the rats were anesthetized with IP ketamine $(100 \mathrm{mg} / \mathrm{kg})$ and then sacrificed by exsanguination from the abdominal aorta. Serum samples were drawn for biochemical assays, and the lung tissues were removed for histopathological studies.

Serum biochemical analyses. Malondialdehyde (MDA) is an aldehyde available in large quantities following lipid peroxidation. It can be determined using the Esterbauer method. ${ }^{8}$ The Griess assay was used to determine nitric oxide (NO) production. ${ }^{9}$

Lung tissue histopathological studies. A pathologist blinded to the groups examined hematoxylin/eosin
(H\&E)-stained lung tissue sections with a light microscope. Lung injury was scored according to alveolar wall thickening and neutrophil infiltration. A score of 0 to 3 points was recorded for each criterion (0: normal, 1: minimal, 2: medium, 3: severe). The total lung injury score (TLIS) was calculated as the sum of these scores. ${ }^{10}$

Statistical analysis. IBM SPSS 20.0 was used to perform descriptive statistical analyses (IBM Corp., Armonk, NY, USA). The Kolmogorov-Smirnov test was applied to test for a normal distribution. The Kruskal-Wallis test was used to evaluate the data. The variables with statistically significant differences were evaluated with the Mann-Whitney $U$ test. The results were presented as mean \pm standard error. Results with a p-value less than 0.05 were considered statistically significant.

Results. Serum MDA and NO levels were significantly higher in group IRI $(p=0.001)$ than in group C $(p=0.006)$. The serum MDA and NO levels were significantly lower in groups IRICO and IRICOD than in group IRI (for MDA; $p=0.005$ and $p=0.002$, and for NO; $p=0.011$ and $p=0.008$, respectively) (Table 1). There were higher alveolar wall thickening and neutrophil infiltration in group IRI $(p=0.001)$ than in group $\mathrm{C}(p<0.001)$. Co-administration of desflurane and CO significantly decreased alveolar wall thickening and neutrophil infiltration $(p=0.006)$ compared to group IRI $(p=0.014)$. Total lung injury score were significantly higher in groups IRI $(p<0.001)$, IRID $(p=0.010)$ and IRICO $(p=0.025)$ than in group C. Total lung injury scores were significantly lower in groups IRID $(p=0.010)$, IRICO $(p=0.004)$, and IRICOD $(p<0.001)$ than in group IRI (Table 2, Figure 1).

Discussion. Lung injury can frequently occur as a result of IRI occurring in distant organs. For example, thoracoabdominal aortic aneurysm repairs can lead to an incidence of lung injury varying between $20-40 \%$. Experimentally, lung injury occurs in response to lower

Table 1 - Serum biochemical analyses.

\begin{tabular}{|c|c|c|c|c|c|c|}
\hline Compound & $\underset{(n=6)}{\text { Group } C}$ & $\begin{array}{l}\text { Group IRI } \\
(\mathrm{n}=6)\end{array}$ & $\begin{array}{c}\text { Group IRID } \\
(\mathrm{n}=6)\end{array}$ & $\underset{(\mathrm{n}=6)}{\text { Group IRICO }}$ & $\underset{(\mathrm{n}=6)}{\text { Group IRICOD }}$ & P-value ${ }^{\ddagger}$ \\
\hline $\operatorname{MDA}(\mathrm{nmol} / \mathrm{mL})$ & $3.4 \pm 1.4$ & $28.3 \pm 6.8^{*}$ & $14.0 \pm 4.3$ & $6.9 \pm 2.0 \dagger$ & $4.4 \pm 0.3 \dagger$ & 0.001 \\
\hline $\mathrm{NO}(\mu \mathrm{mol} / \mathrm{L})$ & $16.4 \pm 6.9$ & $80.5 \pm 12.4^{*}$ & $33.6 \pm 4.6$ & $21.3 \pm 4.2 \dagger$ & $19.0 \pm 3.55 \dagger$ & 0.003 \\
\hline
\end{tabular}

Values are presented as mean \pm SD. ${ }^{\ddagger}$ Significance level with Kruskal Wallis test $\mathrm{p}<0.05,{ }^{*} \mathrm{p}<0.05$ : Compared to group $\mathrm{C}, \mathrm{p}<0.05$ : Compared to ischemia-reperfusion injury group. MDA: malondialdehyde, NO: nitric oxide 
Table 2 - Lung tissue histopathological results.

\begin{tabular}{|c|c|c|c|c|c|c|}
\hline Results & $\underset{(n=6)}{\text { Group } C}$ & $\underset{\substack{\text { Group IRI } \\
(n=6)}}{\text { Ge }}$ & $\underset{(n=6)}{\text { Group IRID }}$ & $\underset{(n=6)}{\text { Group IRICO }}$ & $\underset{(n=6)}{\text { Group IRICOD }}$ & $P$-value ${ }^{\ddagger}$ \\
\hline Neutrophil infiltration & $0.33 \pm 0.21$ & $2.17 \pm 0.31^{*}$ & $1.33 \pm 0.21$ & $1.00 \pm 0.26$ & $0.67 \pm 0.33 \dagger$ & 0.001 \\
\hline Alveolar wall thickening & $0.33 \pm 0.21$ & $1.83 \pm 0.31^{*}$ & $1.00 \pm 0.00$ & $1.16 \pm 0.17$ & $0.83 \pm 0.21 \dagger$ & $<0.001$ \\
\hline Total score & $0.67 \pm 0.33$ & $4.00 \pm 0.26^{*}$ & $2.33 \pm 0.21^{*} \dagger$ & $2.17 \pm 0.31^{*} \dagger$ & $1.50 \pm 0.43 \dagger$ & $<0.001$ \\
\hline
\end{tabular}

Values are presented as mean \pm SE. ${ }^{\ddagger}$ Significance level with Kruskal Wallis test $p<0.05,{ }^{*} p<0.05$ : compared to group C, $\dagger p<0.05$ compared to group ischemia-reperfusion injury

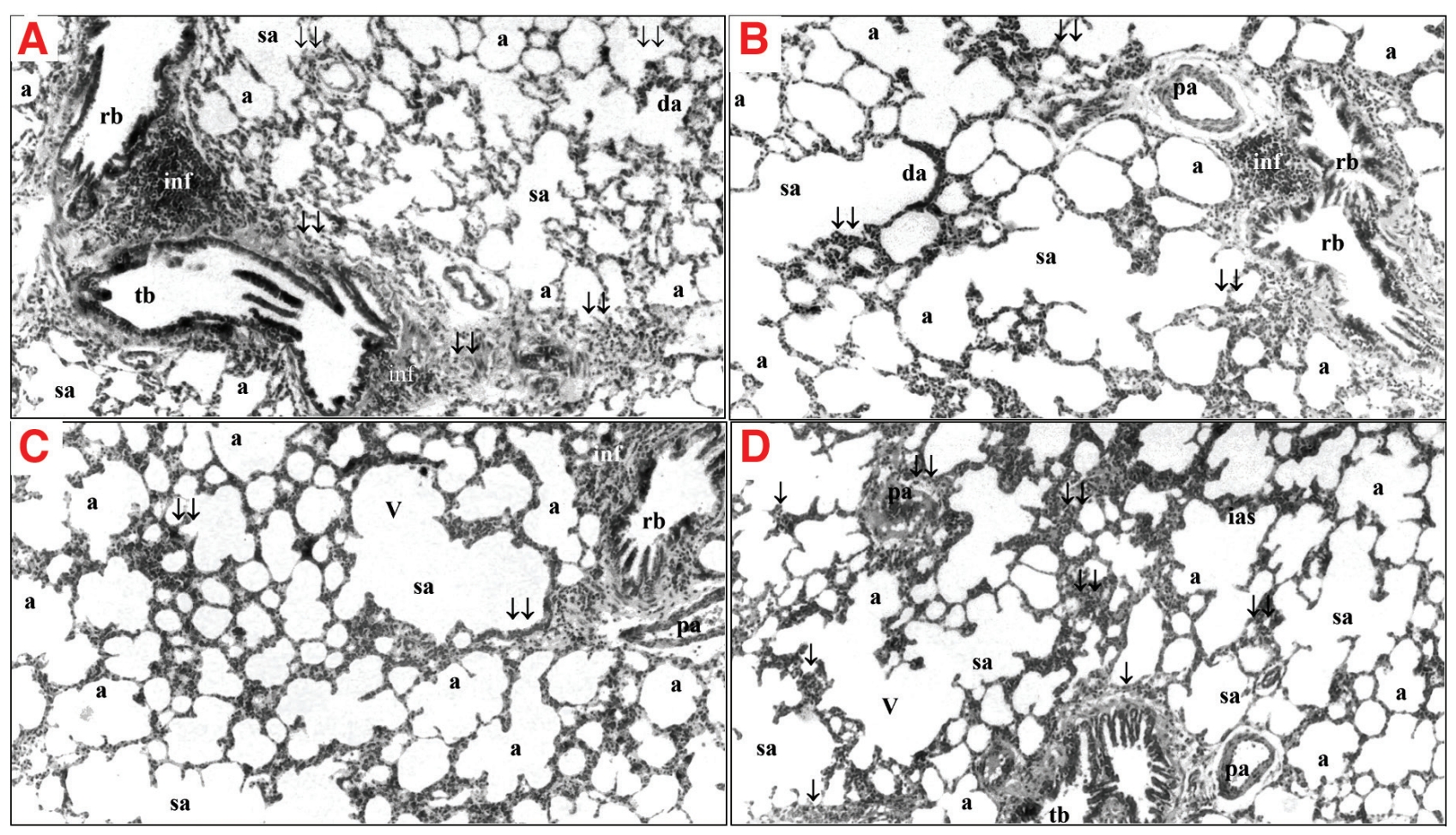

Figure 1 - Lung histology (hematoxylin and eosin staining). A) severe neutrophilic infiltration and increase in alveolar wall thickening, group IRI; B) minimal-medium neutrophilic infiltration and increase in alveolar wall thickening, group IRID; C) minimal neutrophilic infiltration and increase in alveolar wall thickening, group IRICO; D) minimal neutrophilic infiltration and increase in alveolar wall thickening, group IRICOD; H\&E, $\times 40$. a: alveolus, tb: terminal bronchioles, rb: respiratory bronchioles, sa: saccus alveolaris, da: ductus alveolaris, pa: pulmonary artery, V: vacuolization, ias: interalveolar septum, inf: inflammation, $\downarrow$ : pulmonary vascular thickening, $\downarrow \downarrow$ : alveolar wall thickening

extremity IRI when the alveolar epithelium and the capillary endothelium are affected. ${ }^{11}$

Our findings support the existence of systemic oxidative stress due to lower extremity IRI and occurrence of lung injury. Therefore, our finding that intraperitoneally administered $\mathrm{CO}$ can reduce oxidative stress and decrease the lung injury caused by IRI has potential clinical relevance. Interestingly, desflurane alone did not yield similar protective effects to those obtained with $\mathrm{CO}$, but co-administration of $\mathrm{CO}$ with desflurane resulted in a further reduction in the oxidative stress and injury in the lungs than was observed with $\mathrm{CO}$ alone. In recent years, $\mathrm{CO}$ has been the subject of experimental studies as a potential antioxidant therapeutic agent. However, reliable human health data are not yet available, and some experimental studies have shown that $\mathrm{CO}$ can also cause oxidative damage. Therefore, we investigated the IP application of CO and evaluated its efficacy in the IRI rat model. We preferred the IP route to intravenous route because of its ease of application and because it provides a rapid absorption. ${ }^{12}$ 
Cerium oxide has been shown to be effective in smoking related oxidative stress, hepatotoxicity, neurodegenerative diseases, retinopathy, cardiomyopathy, obesity, and intestinal IR injury. ${ }^{4,13}$ However, published data regarding lung protection by $\mathrm{CO}$ are scarce. We previously reported that $\mathrm{CO}$ reduced oxidative damage in lung tissue following non-pulmonary IRI. ${ }^{7}$ Similarly, Serebrovskaa et al investigated the lung protective effect of $\mathrm{CO}$ in experimental pneumonia and observed reduced severity in inflammation on the lung tissues of rats treated with CO. Their results revealed significantly lower levels of ROS in the CO administered rats. Their findings supported the lung protective effects of CO ${ }^{14}$ Consistent with the previous results by Serebrovskaa et al, we also showed significant reductions in inflammation based on histological findings and in oxidative damage based on MDA and NO levels when rats were administered CO.

Experimental and clinical studies have focused on isoflurane and sevoflurane, and the data regarding the antioxidant activity of desflurane are equivocal. In fact, there are studies showing that desflurane increases oxidative damage. ${ }^{3,4}$ We have shown that when desflurane was administered alone, it suppressed the increases in MDA and NO levels induced by IR injury, although the differences were not significant. These conflicting results may reflect differences in desflurane application concentrations and the flow rates at which it is applied, as suggested by the work of Kantekin et al. ${ }^{15}$

Administration of desflurane or CO individually resulted in no significant improvement in alveolar wall thickening or neutrophil infiltration. However, TLISs showed a significant improvement in cases where either agent was administered individually. However, no significant improvement in oxidative damage was shown in rats when desflurane was applied alone, and this may again reflect the contradictory results in the literature regarding the antioxidant activity of desflurane. Nevertheless, CO alone also did not provide a significant improvement in neutrophil infiltration and alveolar wall thickening, whereas evaluation of the histopathological score and the oxidative damage indicated that $\mathrm{CO}$ did have a protective effect. By contrast, evaluation of rats in which desflurane and $\mathrm{CO}$ were administered together revealed that this combination improved histopathology and prevented oxidative damage to a great extent than when both agents were administered alone.

In conclusion, $\mathrm{CO}$ administered intraperitoneally at a dose of $0.5 \mathrm{mg} / \mathrm{kg} 30$ minutes before ischemia in rats anesthetized with desflurane reduced oxidative stress and corrected the lung damage caused by IRI. Desflurane, suppressed oxidative damage in lung tissue after IRI, even though statistical significance was not reached. As an innovation, we showed that this antioxidant activity can become stronger when used with cerium oxide. We think that $\mathrm{CO}$ administration before IRI and desflurane anesthesia during IRI can have a lung protective effect in rats. These findings implicate an organ-protective effect of cerium oxide during desflurane anesthesia. So the protective effects of this nanoparticle in different organs during desflurane anesthesia can also be demonstrated in future experimental studies. More studies are needed to support the transfer of this promising therapeutic option to clinical practice.

Study limitations. One limitation is the absence of administering CO intravenously although it has similar biodistribution to that achieved with IP administration. The other limitation is that CO was only applied simultaneously with clamping of the aorta. Thus, we did not studied its effects when administered following IRI. Another limitation was that we did not calculate sample size. The number of rats was determined in accordance with the recommendations of the animal research committee for the welfare of animals. Additionally, since all animals were anesthetized with ketamine and xylazine, the effects of ketamine and xylazine on oxidative damage could not be excluded. However, these anesthetics administered for the welfare of the animals were administered to all groups in equal doses at the beginning of the experiment, and this limitation was tried to be avoided.

Received 11th July 2021. Accepted 25th August 2021.

From the Department of Anesthesiology and Reanimation (Ozdemirkan, Arslan, Sivgin), Gazi University Faculty of Medicine, from the Department of Medical Biochemistry (Boyunaga), Ankara Medipol University, Ankara, from the Department of Physiology (Kucuk), Faculty of Medicine, Kütahya Health Science University, Kütahya, from the Department of Anesthesiology and Reanimation (Gunes), from the Department of Cardiovascular Surgery (Tuncay), Faculty of Medicine, Erciyes University, Kayseri, and from the Department of Histology and Embryology (Sezen), Faculty of Medicine, Kirlkkale University, Kirlkkale, Turkey.

Address correspondence and reprints request to: Dr. Mustafa Arslan, Department of Anesthesiology and Reanimation, Gazi University Hospital, Ankara, Turkey. E-mail:mustarslan@gmail.com

ORCID ID: http://orcid.org/0000-0003-4882-5063

\section{References}

1. Takhtfooladi HA, Hesaraki S, Razmara F, Takhtfooladi MA, Hajizadeh $\mathrm{H}$. Effects of $\mathrm{N}$-acetylcysteine and pentoxifylline on remote lung injury in a rat model of hind-limb ischemia/ reperfusion injury. J Bras Pneumol 2016; 42: 9-14.

2. Xue B, Chen B, Tang Y, Weng C, Lin L. Dexmedetomidine protects against lung injury induced by limb ischemiareperfusion via the TLR4/MyD88/NF-кB pathway. Kaohsiung J Med Sci 2019; 35: 672-678. 
3. Stevens JL, Feelisch M, Martin DS. Perioperative Oxidative Stress: The Unseen Enemy. Anesth Analg 2019; 129: 1749-1760.

4. Strosing KM, Faller S, Gyllenram V, Engelstaedter H, Buerkle $\mathrm{H}$, Spassov S, et al. Inhaled anesthetics exert different protective properties in a mouse model of ventilator-induced lung injury: Anesthesia \& Analgesia 2016; 123: 143-151.

5. Tatar T, Polat Y, Comu FM, Kartal H, Arslan M, Kucuk A. Effect of cerium oxide on erythrocyte deformability in rat lower extremity ischemia reperfusion injury. Bratisl Lek Listy 2018; 119: 441-443.

6. Gubernatorova EO, Liu X, Othman A, Muraoka WT, Koroleva EP, Andreescu S, et al. Europium-Doped cerium oxide nanoparticles limit reactive oxygen species formation and ameliorate intestinal ischemia-reperfusion injury. Adv Healthc Mater 2017; 6: doi: 10.1002/adhm.201700176.

7. Tuncay A, Sivgin V, Ozdemirkan A, Sezen SC, Boyunaga H, Kucuk A, et al. The effect of cerium oxide on lung tissue in lower extremity ischemia reperfusion injury in sevoflurane administered rats. Int J Nanomedicine 2020; 15: 7481-7489.

8. Esterbauer H, Cheeseman KH. Determination of aldehydic lipid peroxidation products: Malonaldehyde and 4-hydroxynonenal. In: Methods in Enzymology [Internet]. Int J Nanomedicine 2020; 15: 7481-7489.

9. Schmölz L, Wallert M, Lorkowski S. Optimized incubation regime for nitric oxide measurements in murine macrophages using the Griess assay. J Immunol Methods 2017; 449: 68-70.
10. Li D, Sun T, Chi L, Zhao D, Li W. Acupoint catgut embedding improves the lipopolysaccharide-induced acute respiratory distress syndrome in rats. Bio Med Research International 2020; 2020: 1-9.

11. Huwae TECJ, Santoso ARB, Kesuma W, Sujuti H, Ratnawati $\mathrm{R}$, Sukmajaya WP, et al. Reperfusion interval as a prevention of lung injury due to limb ischemia-reperfusion after application of tourniquet in murine experimental study. Indian J Orthop 2020; 54: 704-710.

12. Al Shoyaib A, Archie SR, Karamyan VT. Intraperitoneal route of drug administration: should it be used in experimental animal studies? Pharm Res 2020; 37: 12.

13. Nelson B, Johnson M, Walker M, Riley K, Sims C. Antioxidant cerium oxide nanoparticles in biology and medicine. Antioxidants (Basel) 2016; 5: 15.

14. Serebrovska Z, Swanson RJ, Portnichenko V, Shysh A, Pavlovich $S$, Tumanovska L, et al. Anti-inflammatory and antioxidant effect of cerium dioxide nanoparticles immobilized on the surface of silica nanoparticles in rat experimental pneumonia. Biomed Pharmacother 2017; 92: 69-77.

15. Ünal Kantekin Ç, Çakirca M, Yaman F, Göçmen AY. Does the Application of Desflurane at The Same Concentration by Different Flows Change the Oxidative Stress? Animal Experiment. Online Turkish Journal of Health Sciences 2019; 4: 492-505. 